\title{
INCIDÊNCIA DE INFECÇÕES DO TRATO URINÁRIO EM GESTANTES E CORRELAÇÃO COM O TEMPO DE DURAÇÃO DA GESTAÇÃO
}

\author{
Samara Pavan da VEIGA ${ }^{1}$, Veridiana Lenartovicz BOEIRA ${ }^{2}$, Claudinei Mesquita da SILVA ${ }^{3} \&$ Leyde \\ Daiane de PEDER ${ }^{3 *}$
}

1 Laboratório Municipal, Secretaria Municipal de Saúde. Marmeleiro, Paraná, Brasil.

2 Universidade Estadual do Oeste do Paraná, Cascavel, Paraná, Brasil.

3 Centro Universitário da Fundação Assis Gurgacz, Cascavel, Paraná, Brasil.

*Autor para correspondência: leydepeder@yahoo.com.br

DOI: http://dx.doi.org/10.18571/acbm.125

\section{RESUMO}

As infecções do trato urinário são frequentes durante o período gestacional e as complicações se associam a significativos problemas. $\mathrm{O}$ estudo objetivou verificar a incidência de infecções do trato urinário durante a gestação e a correlação com a duração do período gestacional identificando complicações com o parto prematuro e o baixo peso ao nascer. Trata-se de um estudo retrospectivo com análise de Declarações de Nascidos Vivos e análise de laudos laboratoriais de exame de urina I de gestantes atendidas pelo Laboratório Municipal de Marmeleiro - PR, durante o período de pré-natal. A incidência de infecção do trato urinário encontrada foi de $10,1 \%$ e dentre essas, 18,2\% tiveram parto prematuro. Das que tiveram parto prematuro, 63,6\% não apresentavam os 3 exames de urina I preconizados pelo Programa Rede Mãe Paranaense; 10,4\% dos bebês apresentaram baixo peso ao nascer e 33,3\% nasceram de períodos gestacionais com incidência de infecção urinária. A partir dos resultados concluiu-se que existe correlação entre a incidência de infecções do trato urinário na gestação e o aumento das taxas de prematuridade.

Palavras-chave: Infecções do trato urinário; Gestação; Parto prematuro; Baixo peso ao nascer.

\begin{abstract}
Urinary tract infections are frequent during the gestational period and complications are associated with significant problems. The study aimed to verify the incidence of urinary tract infections during gestation and the correlation with gestational period duration, identifying premature delivery and low birth weight. This is a retrospective study with analysis of Declarations of Live Births and analysis of laboratory reports of urine examination I of pregnant women attended by the Municipal Laboratory of Marmeleiro - PR, during the prenatal period. The incidence of urinary tract infections was $10.1 \%$ and among these, $18.2 \%$ had a preterm birth. Of those who had preterm delivery, $63.6 \%$ did not present the 3 urine exams recommended by the Rede Mãe Paranaense Program; $10.4 \%$ of the infants presented low birth weight and $33.3 \%$ were born from gestational periods with an incidence of urinary tract infection. From the results it was concluded that there is a correlation between the incidence of urinary tract infections during pregnancy and the increase in the rates of prematurity.
\end{abstract}

Keywords: Urinary tract infections; Pregnancy; Premature; Low birth weight.

\section{Introdução}

As infecções do trato urinário (ITU) são muito frequentes durante o período gestacional, chegando a ser a terceira ocorrência clínica mais comum devido às mudanças anatômicas e fisiológicas do trato urinário, que ocorrem durante esse período (NASCIMENTO et al, 2012). De 


\section{Biomedica Brasiliensia}

acordo com MATA et al (2014), as complicações desses casos ocorrem em cerca de $20 \%$ das gestações e são responsáveis por $10 \%$ dos internamentos durante a gravidez, associando-se a significativos problemas relacionados à morbimortalidade materna e perinatal. Dentre as complicações destacam-se o trabalho de parto e parto pré-termo, ruptura prematura de membranas amnióticas, restrição de crescimento intrauterino, recém-nascidos de baixo peso e óbito perinatal (DUARTE et al, 2002; DARZÉ et al, 2011). Além da incidência aumentada dessas infecções entre grávidas, é justamente neste período que o arsenal terapêutico antimicrobiano e as possibilidades profiláticas são mais restritas, considerando-se a toxicidade das drogas para o feto (DUARTE et al, 2002; GÓMEZ, 2015).

De acordo com Paganoti et al. (2015), a prematuridade está relacionada à alta morbidade e mortalidade neonatais, ao alto custo nos cuidados a esses recém-nascidos e à dificuldade em se estabelecer medidas eficazes capazes de prevenir tal evento, o que constitui um importante problema de saúde pública em nosso país, já que, segundo dados do Sistema Nacional de Nascidos Vivos, em pesquisa liderada pelo Programa de Pós-Graduação em Epidemiologia da Universidade Federal de Pelotas, o Brasil apresentou uma taxa de prematuridade de 11,8\% no ano de 2011. Diversos estudos vêm demonstrando um discreto, porém, crescente aumento ao longo do tempo, contrariando o que seria esperado em um país no qual a maioria dos indicadores de saúde maternoinfantil vem apresentando melhorias (UNICEF, 2013).

No sentido de diminuir a incidência de complicações materno-fetais, garantir a qualidade da atenção pré-natal e reduzir os indicadores de mortalidade materna e infantil, o estado do Paraná implantou o programa Rede Mãe Paranaense, que visa uma série de ações, tais como a captação precoce da gestante e o seu acompanhamento ${ }^{8}$. Seguindo a linha guia deste programa, se estabelece um fluxo de consultas e exames a serem realizados, assegurando à mulher o direito ao planejamento reprodutivo e atenção humanizada à gravidez, ao parto e ao puerpério; e à criança, é garantido o direito ao nascimento seguro e ao crescimento e desenvolvimento saudáveis ${ }^{9}$.

Dentre os diversos exames preconizados pelo programa, encontra-se a realização do exame de urina I nos três trimestres de gestação, tendo em vista a grande incidência de intercorrências clínicas associadas às infecções do trato urinário e aos problemas que podem desencadear ao longo do período gestacional (Linha Guia da Rede Mãe Paranaense, 2013).

O estudo deste tema é de grande importância em função da elevada incidência de ITU no período gestacional e dos impactos sobre a saúde da mulher e do feto e, portanto, a qualidade da atenção pré-natal é fundamental para o diagnóstico e tratamento precoce, assim como, melhora do prognóstico materno-fetal. Diante do exposto, esse estudo teve como objetivo verificar a incidência de infecções do trato urinário durante o período gestacional e verificar se existe correlação ou não com a duração do período gestacional, identificando complicações como o parto prematuro e o baixo peso ao nascer.

\section{Material e métodos}

Estudo retrospectivo de caráter descritivo com abordagem quantitativa, realizada através da análise de todas as declarações de nascidos vivos (DN), recebidas e arquivadas pela Secretaria Municipal de Saúde, de mães residentes no município de Marmeleiro - PR, que tiveram data do parto realizada no período de $1^{\circ}$ de janeiro a 31 de dezembro de 2015 e análise de laudos laboratoriais de exame de urina I de gestantes atendidas pelo Laboratório Municipal de Marmeleiro - PR, durante o período de pré-natal.

Foi considerado como período de pré-natal, aquele compreendido a partir da realização do exame para confirmação da gestação pelo próprio laboratório municipal até a data do parto, constante na DN. Para as gestantes que não possuíam resultado laboratorial positivo realizado pelo próprio Laboratório Municipal, foram considerados os exames de urina I realizados a partir da $1^{\mathrm{a}}$ coleta de pré-natal, que também acompanhavam a realização de exames de glicemia de jejum, 
teste triagem para sífilis e tipagem sanguínea, dentre outros, conforme protocolo da Rede Mãe Paranaense (Linha Guia da Rede Mãe Paranaense, 2013).

As variáveis coletadas das DN foram iniciais do nome da mãe (gestante), idade da mãe, tempo de duração da gestação, mês de gestação em que o pré-natal foi iniciado e peso do bebê ao nascer. Após, foi realizada a busca de todos os laudos laboratoriais das gestantes em estudo, a fim de identificar quais realizaram seus exames de pré-natal no Laboratório Municipal de Marmeleiro e coletar os resultados relativos à presença de reação nitrito positivo (detectado pela tira de análise química), a partir da urinálise, que somente é positiva na presença de enterobactérias (PAULA et al, 2015), um dos principais agentes causadores de infecções do trato urinário (D'ADDAZIO e MORAES, 2015).

Foram excluídos da pesquisa, os dados de DN com data do parto realizada fora do período estabelecido para o estudo, de gestantes que não residiam no município e gestantes que não realizaram exames de urina I durante o período de pré-natal no Laboratório Municipal de Marmeleiro - PR.

Para o estudo, as pacientes foram divididas em dois grupos, aquelas que apresentaram incidência de ITU com reação nitrito positivo e as que não apresentaram incidência de ITU com reação nitrito positivo durante o período de pré-natal.

Os dados coletados foram armazenados em Microsoft Excel $^{\circledR}$ e para análise e estudo sobre a associação entre variáveis utilizou-se estatística descritiva e adotou-se o teste de Qui Quadrado em nível de significância de 5,0\%, com resultados considerados estatisticamente significativos para $\mathrm{p} \leq 0,05$. A análise estatística foi realizada através do software Bioestat ${ }^{\circledR}$.

O trabalho foi aprovado pelo Comitê de Ética em Pesquisa com Seres Humanos do Centro Universitário da Fundação Assis Gurgacz, aprovado em 22 de agosto de 2016, sob parecer número 1.690.058, e sob o cumprimento de todos os procedimentos metodológicos da resolução 466/12 do Conselho Nacional de Saúde.

\section{Resultados}

Foram analisadas inicialmente, 204 declarações de nascidos vivos, as quais referiam-se a 202 gestantes, uma vez que foram verificadas 2 gestações de gemelares. Foram excluídas 40 DN, que se referiam a 39 gestantes que não atendiam aos critérios de inclusão estabelecidos para o estudo.

As características das 163 gestantes incluídas no estudo constam na Tabela 1. Verificouse que $72,4 \%$ (118) possuíam idade entre 21 e 40 anos, em 93,3\% (152) a duração da gestação variou entre 37 e 41 semanas e 6,7\% (11) tiveram parto pré-termo. Entre as 11 gestantes que tiveram parto pré-termo, e apresentaram significância estatística com $p \leq 0,05,63,6 \%$ (7) estavam no grupo das gestantes com menos de 3 exames de urina realizados, conforme preconizado pelo Programa Rede Mãe Paranaense.

Em relação ao período de início do pré-natal, 81,6\% (133) declararam ter iniciado ainda no $1^{\circ}$ trimestre gestacional.

Dentre as 54 gestantes que tiveram menos de 3 exames de urina, 37\% (20) haviam informado que iniciaram o pré-natal após o $1^{\circ}$ trimestre gestacional e $13 \%$ (7) resultaram em parto prematuro, apresentando significância estatística com $\mathrm{p} \leq 0,05$. 
Tabela 1: Variáveis coletadas das DN de acordo com o número de exames de urina realizados pelo Laboratório Municipal de Marmeleiro, Paraná, Brasil, 2017.

\begin{tabular}{|c|c|c|c|c|c|}
\hline Caracterís & cas & Total n (\%) & $\begin{array}{c}\text { Mulheres com } \\
\text { pelo menos } 3 \\
\text { exames durante } \\
\text { a gestação } \\
\text { n }(\%)\end{array}$ & $\begin{array}{c}\text { Mulheres com } \\
\text { menos de } 3 \\
\text { exames } \\
\text { durante a } \\
\text { gestação } \\
\text { n (\%) }\end{array}$ & Valor de $p^{*}$ \\
\hline $\begin{array}{l}\text { Idade da mãe } \\
\text { (Anos) }\end{array}$ & $\begin{array}{c}\text { Até } 20 \\
21-30 \\
31-40 \\
\geq 41\end{array}$ & $\begin{array}{c}44(27,0) \\
84(51,5) \\
34(20,9) \\
01(0,6)\end{array}$ & $\begin{array}{c}29(26,6) \\
59(54,1) \\
20(18,4) \\
01(0,9)\end{array}$ & $\begin{array}{c}15(27,8) \\
25(46,3) \\
14(25,9) \\
0(0)\end{array}$ & 0,585 \\
\hline $\begin{array}{l}\text { Duração da } \\
\text { gestação } \\
\text { (Semanas) }\end{array}$ & $\begin{array}{l}22 \text { a } 27 \\
28 \text { a } 36 \\
37 \text { a } 41\end{array}$ & $\begin{array}{c}2(1,2) \\
9(5,5) \\
152(93,3)\end{array}$ & $\begin{array}{c}0(0) \\
4(3,7) \\
105(96,3)\end{array}$ & $\begin{array}{c}2(3,7) \\
5(9,3) \\
47(87,0)\end{array}$ & 0,040 \\
\hline $\begin{array}{l}\text { Trimestre que } \\
\text { iniciou o pré-natal }\end{array}$ & $\begin{array}{c}1^{\circ} \\
\text { Após o } 1^{\circ}\end{array}$ & $\begin{array}{l}133(81,6) \\
30(18,4)\end{array}$ & $\begin{array}{c}99(90,8) \\
10(9,2)\end{array}$ & $\begin{array}{l}34(63,0) \\
20(37,0)\end{array}$ & $<0,0001$ \\
\hline
\end{tabular}

Analisando-se a Tabela 2 que identifica o peso ao nascer das 164 declarações de nascidos vivos incluídas no estudo, 10,4\% dos bebês (17) apresentavam-se com menos de 2,500 gramas, sendo classificados como baixo peso ao nascer (BPN).

Tabela 2: Peso da criança ao nascer a partir das DN, de acordo com o número de exames de urina realizados pelo Laboratório Municipal de Marmeleiro, Paraná, Brasil, 2017.

\begin{tabular}{|c|c|c|c|c|}
\hline Características & $\begin{array}{l}\text { Total } \\
\text { n (\%) }\end{array}$ & $\begin{array}{c}\text { Mulheres com } \\
\text { pelo menos } 3 \\
\text { exames } \\
\text { durante a } \\
\text { gestação } \\
\text { n }(\%)\end{array}$ & $\begin{array}{c}\text { Mulheres com } \\
\text { menos de } 3 \text { exames } \\
\text { durante a gestação } \\
\text { n }(\%)\end{array}$ & $\begin{array}{c}\text { Valor de } \\
p^{*}\end{array}$ \\
\hline $\begin{array}{cc} & <2500 \\
\text { Peso do bebê ao } & 2501 \text { a } 2999 \\
\text { nascer (gramas) } & 3000 \text { a } 3999 \\
& \geq 4000\end{array}$ & $\begin{array}{c}17(10,4) \\
41(25,0) \\
100(61,0) \\
6(3,6)\end{array}$ & $\begin{array}{c}12(10,91) \\
26(23,64) \\
67(60,91) \\
5(4,54)\end{array}$ & $\begin{array}{c}5(9,3) \\
15(27,8) \\
33(61,1) \\
1(1,8)\end{array}$ & 0,786 \\
\hline
\end{tabular}

$\mathrm{n}$ - número de pacientes; *Teste de Qui quadrado foi utilizado para significância estatística para $\mathrm{p} \leq 0,05$.

Após a busca e análise dos laudos laboratoriais emitidos pelo Laboratório Municipal de Marmeleiro a partir das gestantes apresentadas nas Declarações de Nascidos Vivos, verificou-se que 109 gestantes realizaram pelo menos 3 exames de urina, conforme preconiza o guia da Rede Mãe Paranaense. Nestas, $11(10,1 \%)$ foram diagnosticadas como portadoras de infecção urinária ao menos 1 vez durante o período gestacional, devido à presença de reação positiva para o teste do nitrito na fita reagente, e dentre essas, $18,2 \%$ (2) dos casos tiveram parto realizado antes de 37 semanas de gestação (Tabela 3). 
Tabela 3: Relação entre incidência de infecção urinária em gestantes com pelo menos 3 exames de urina I e correlação com o tempo de duração da gestação. Marmeleiro, Paraná, Brasil, 2017.

\begin{tabular}{|c|c|c|c|c|c|}
\hline \multicolumn{6}{|c|}{ Incidência de Infecção Urinária } \\
\hline Características & & $\begin{array}{c}\text { Sim } \\
\text { n (\%) }\end{array}$ & $\begin{array}{l}\text { Não } \\
\text { n (\%) }\end{array}$ & $\begin{array}{l}\text { Total } \\
\text { n (\%) }\end{array}$ & Valor de $p^{*}$ \\
\hline \multirow{3}{*}{$\begin{array}{c}\text { Duração da } \\
\text { gestação } \\
\text { (semanas) }\end{array}$} & 28 a 36 & $2(18,2)$ & $2(2,0)$ & $4(3,7)$ & 0,063 \\
\hline & 37 a 41 & $9(81,8)$ & $96(98,0)$ & $105(96,3)$ & \\
\hline & Total & $11(10,1)$ & $98(89,9)$ & $109(100,0)$ & \\
\hline
\end{tabular}

Observou-se também que 12 declarações de nascidos vivos, presentes no grupo com 3 ou mais exames de Urina I, apresentavam crianças com menos de 2,500 gramas, consideradas como baixo peso ao nascer, sendo que 33,3\% (4) foram oriundas de períodos gestacionais com incidência de ao menos 1 episódio de infecção urinária com reação nitrito positivo (Tabela 4), apresentando significância estatística com $\mathrm{p} \leq 0,05$.

Tabela 4. Relação entre incidência de infecção urinária em gestantes com pelo menos 3 exames de urina e correlação com o peso ao nascer da criança. Marmeleiro, Paraná, Brasil, 2017.

\begin{tabular}{|c|c|c|c|c|c|}
\hline \multicolumn{6}{|c|}{ Incidência de Infecção Urinária } \\
\hline Características & & $\begin{array}{c}\text { Sim } \\
\text { n (\%) }\end{array}$ & $\begin{array}{l}\text { Não } \\
\text { n (\%) }\end{array}$ & $\begin{array}{l}\text { Total } \\
\text { n (\%) }\end{array}$ & Valor de $p^{*}$ \\
\hline Peso da criança & $<2500$ & $4(33,3)$ & $8(8,2)$ & $12(10,9)$ & 0,031 \\
\hline $\begin{array}{l}\text { ao nascer } \\
\text { (Gramas) }\end{array}$ & $\geq 2500$ & $8(66,7)$ & $90(91,8)$ & $98(89,1)$ & \\
\hline Total & & $12(100,0)$ & $98(100,0)$ & $110(100,0)$ & \\
\hline
\end{tabular}

\section{Discussão}

O município de Marmeleiro, situado na região sudoeste do estado do Paraná, com uma população estimada de 13.909 habitantes conforme dados do IBGE de 2010 (IBGE, 2010), e com economia voltada estritamente para o agronegócio, faz parte de um grupo de 27 municípios sob a área de abrangência da $8^{\text {a }}$ Regional de Saúde de Francisco Beltrão - macrorregional oeste. Essa regionalização dos serviços de saúde no estado do Paraná foi se desenvolvendo ao longo do tempo, tendo como base a organização política municipal, a oferta de serviços de saúde e os polos regionais de desenvolvimento (Secretaria de Saúde do Estado do Paraná, 2016).

Segundo dados da Secretaria Estadual de Saúde, o Paraná vem apresentando uma série de ações e estratégias a fim de diminuir as taxas de prematuridade no estado. Como parte destas ações, o programa Rede Mãe Paranaense vem apresentando consideráveis avanços na área da atenção materno-infantil apresentando uma média de 10,2\% de nascimentos prematuros entre os anos de 2013 a 2016 e mantendo esses índices abaixo da prevalência nacional de 11,3\%, segundo dados do Estudo Nacional de Base Hospitalar realizado em 2013 (Secretaria de Saúde do Estado do Paraná, 2016).

Visando analisar a incidência de ITU e sua correlação com o tempo de duração de gestação, este estudo também buscou identificar possíveis complicações que essa associação pode resultar, 


\section{Biomedica Brasiliensia}

como o parto prematuro e o baixo peso ao nascer.

No cenário das 163 gestantes inicialmente analisadas a partir das 164 DN, 72,4\% (118) delas tinha idade entre 21 e 40 anos e $27 \%$ (44) tinham menos de 20 anos, números muito próximos aos encontrados em outro estudo realizado no município de Francisco Beltrão, ambos localizados na mesma região do estado do Paraná, no ano de 2015 , que mostrou que $82 \%$ das gestantes estudadas tinham entre 15 e 35 anos (COSTA et al, 2016), concordando também com outro estudo realizado no estado de São Paulo, onde verificou-se que 20,5\% eram adolescentes (10 a 19 anos) e $69,3 \%$ estavam entre 20 e 34 (LAURENTI et al, 2015). Vários estudos relatam que, tanto gestações precoces quanto as gestações tardias, tendem a apresentar uma maior incidência de complicações materno-fetais e que podem resultar em partos prematuros e no baixo peso ao nascer (SILVA e SURITA, 2009), o que pode explicar o fato da maioria das gestações ocorrerem na faixa etária de 21 a 40 anos.

Por serem consideradas como gestações de risco, ocorre também a baixa incidência de casos de mães com idades mais elevadas, embora alguns estudos demonstrem, que devido aos avanços da medicina, as gestações nessa faixa etária vem aumentando mundialmente (GONÇALVES e MONTEIRO, 2012).

Em relação à idade gestacional, 93,3\% (152) tiveram parto realizado entre 37 e 41 semanas, e 6,7\% (11) tiveram parto realizado antes de 37 semanas, definidos, portanto como partos prétermo. Resultado abaixo do encontrado em outros estudos, que revelam taxas de prematuridade entre $9,3 \%{ }^{16}$ e 12,3\% (LAJOS, 2014). Esta última taxa refere-se ao resultado do Estudo Multicêntrico de Investigação em Prematuridade coordenado pela UNICAMP, que também pesquisou os diversos riscos para o parto prematuro, destacando-se a gravidez múltipla e as infecções urinárias (LAJOS, 2014; BETTIOL et al, 2010). De acordo com Machado et al (2016), a escassez de estudos sobre a prematuridade no Brasil, principalmente em municípios de pequeno porte, e a variação das taxas entre as diferentes regiões, dificultam a identificação dos fatores de risco, bem como no estabelecimento de medidas para a sua redução. Essa baixa taxa de prematuridade encontrada também pode representar a importância de se seguir as estratégias propostas pelo programa Rede Mãe Paranaense e reduzir de fato os indicadores de complicações materno-infantis.

Isso também pode ser reforçado pelo dado que nos mostra que dentre os 11 partos prematuros analisados, e apresentando significância estatística com $p \leq 0,05,63,6 \%$ (7) faziam parte do grupo das gestantes que não apresentavam os laudos laboratoriais de pelo menos 3 exames de urina I, conforme preconizado pelo programa Rede Mãe Paranaense, e que pode ser resultado da falha no processo de busca ativa das gestantes que não compareceram ao acompanhamento de exames e consultas na atenção primária à saúde. Isso nos remete também à importância da qualidade do pré-natal ofertado, podendo ser resultado da falta de continuidade da assistência preconizada pelo Sistema Único de Saúde.

Esse problema também pode ser constatado pelas taxas referentes ao período de início de pré-natal. Embora 81,6\% (133) tenham declarado terem iniciado o seu acompanhamento ainda no $1^{\circ}$ trimestre gestacional, $37 \%$ (20) das gestantes que não possuíam pelo menos 3 exames de Urina I preconizados pelo programa declararam ter iniciado o pré-natal tardiamente e 13\% (7) resultaram em parto prematuro, apresentando significância estatística com $p \leq 0,05$. Nesse sentido, a necessidade de se iniciar o pré-natal ainda no $1^{\circ}$ trimestre, favorece a monitoração dos fatores de risco e contribui para a redução dos agravos que podem estar presentes nesse período, uma vez que poderão ser detectados e tratados precocemente (BAGGIO et al, 2016).

Dentre as 109 gestantes que realizaram os 3 exames de urina I preconizados, 10,1\% (11) tiveram ITU com reação nitrito positivo ao menos uma vez durante o período gestacional. Em estudo realizado na região nordeste do Brasil, a prevalência encontrada foi de $12,3 \%$, obtida a partir de exames de uroculturas positivas (DARZÉ et al, 2011). E dentre os 11 casos que tiveram infecção urinária durante o período gestacional, 18,2\% (2) dos casos tiveram parto realizado antes 


\section{Biomedica Brasiliensia}

de 37 semanas de gestação, o que nos mostra a importância da detecção precoce de ITU durante o pré-natal, como forma de antecipar possíveis agravos, que podem influenciar no trabalho de parto prematuro e na saúde da mãe e do bebê.

Essa situação também é reconhecida com fator agravante para a estratificação de gestação de risco, sendo a ITU relatada como fator relevante para complicação do período gestacional, visto que agrava tanto o prognóstico materno quanto o perinatal, revelando taxas de $14,8 \%$ de ITU em gestantes classificadas como alto risco em estudo realizado no município de Francisco Beltrão, estado do Paraná, no ano de 2015 (Costa et al, 2016). Outros estudos revelam que a ITU ocorre em 17 a $20 \%$ das gestações e se associa a complicações graves que aumentam consideravelmente as taxas de morbimortalidade materna e neonatal (BRASIL, 2012).

Nesse contexto, a realização do exame de urina I, que consiste na avaliação de fitas reagentes e análise do sedimento urinário, é fundamental para a caracterização e prognóstico da ITU (NASCIMENTO et al, 2012). Dentre os testes químicos presentes nas fitas reagentes, alguns se revestem de importância pelo seu alto valor indicativo da presença de ITU, como é o caso do teste da atividade redutora de nitrato, que somente é positiva na presença de enterobactérias (PAULA et al, 2015), um dos principais agentes causadores de infecções do trato urinário (D'ADDAZIO e MORAES, 2015), e estimando-se que a Escherichia coli seja a responsável por até $90 \%$ dos casos de infecções do trato urinário (MOURA e FERNANDES, 2010).

Isso também ressalta a importância da realização do exame de urina I para a detecção dos possíveis casos de bacteriúria assintomática, frequentes no período gestacional, tendo a Escherichia coli como principal agente etiológico identificado em $80 \%$ desses casos (MATA et al, 2014), podendo evoluir para a ITU sintomática e resultar nas diversas consequências (PAULA et al, 2014).

Entretanto, o teste de maior valor para a confirmação do diagnóstico de uma ITU é a urocultura, porém, por ser um exame oneroso e não fornecer resultado imediato, acaba por ser pouco aplicado no rastreamento dessas infecções (DARZÉ et al, 2011), deixando essa importância para o exame de urina I, que fornece resultados imediatos para complementar o quadro clínico dos pacientes (PAULA et al, 2014), além de ser um exame de baixo custo e fácil realização (GUERRA et al, 2012). Reforçado pelo fato que, após o diagnóstico clínico da infecção urinária e confirmação com exame de urina I, na maioria dos casos, a instituição do tratamento demanda urgência, sem tempo para a obtenção do resultado da urocultura e antibiograma (DUARTE et al, 2002; DUARTE et al, 2008) a fim de se reduzir as complicações que essas infecções podem causar.

Essa correlação pode ser observada também na linha guia do programa Rede Mãe Paranaense, que deixa a realização da urocultura a critério do médico, garantindo-se a sua realização trimestralmente somente para as gestantes consideradas como nível médio ou alto. Essa estratificação de risco serve para orientar e organizar o fluxo a ser seguido nos diferentes níveis de atenção à saúde e definindo a vinculação da gestante ao pré-natal e ao hospital para o atendimento das suas intercorrências na gestação e no momento do parto. Conforme critérios referentes às características individuais (raça, etnia e idade), sociodemográficas (escolaridade) e de história reprodutiva anterior, bem como a presença de condições clínicas pré-existentes (ex.: diabetes, hipertensão arterial) e intercorrências clínicas (ex.: infecções de repetição do trato urinário, trabalho de parto prematuro) a gestante é classificada como risco habitual, risco intermediário e alto risco (Linha Guia da Rede Mãe Paranaense, 2013).

Em relação ao peso ao nascer das $164 \mathrm{DN}$ analisadas, 10,4\% (17) nasceram com menos de 2.500 gramas. Valor superior aos já encontrados em estudo realizado no estado de São Paulo em 2011, que mostra taxa de 8,2\% de BPN entre os nascidos vivos analisados (LAURENTI et al, 2015).

O baixo peso ao nascer é um dos fatores de grande importância no estudo da morbimortalidade neonatal, e os principais responsáveis por essa condição são o retardo do crescimento intrauterino e a prematuridade. No Brasil, o BPN é representado principalmente por 
recém-nascidos com retardo de crescimento intrauterino e atinge cerca de $10 \%$ de todos os nascidos vivos (Franciotti et al, 2006; FERRAZ e NEVES, 2011; PEDRAZA e CRISTÓVÃO, 2015).

Essa relação pode estar relacionada aos grandes números de cesáreas que se associam á elevados índices de nascimentos pré-termos, uma vez que muitas dessas cesáreas vem ocorrendo sem razão médica aparente (BETTIOL et al, 2010).

Sabe-se hoje que as infecções do trato urinário e suas complicações no período gestacional podem resultar em casos de prematuridade, baixo peso ao nascer e na morte fetal (SALCEDO et al, 2010), e por isso a importância em se detectar precocemente os casos de ITU durante o prénatal, e também na importância em se seguir o preconizado pelo programa Rede Mãe Paranaense e em se ter uma equipe de saúde preparada para detectar tais eventos.

Das complicações causadas pela ITU na gestação, um estudo realizado em Maceió, entre os anos de 2013 e 2014, mostrou que 57,5\% das gestantes desenvolveram como complicação o trabalho de parto prematuro (MATA et al, 2014). Em um estudo realizado no Rio Grande do Sul, evidenciou-se que a prematuridade esteve presente em $78 \%$ dos nascimentos de crianças com BPN. Quanto mais curta a gestação, menor o tamanho do recém-nascido e maior o risco de mortalidade, morbidade e incapacidade (FERRAZ e NEVES, 2011; PEDRAZA, 2014).

Das 12 crianças com BPN, 33,3\% eram oriundas de gestações com pelo menos 1 episódio de ITU com reação nitrito positivo, detectados durante o exame de urina, durante o pré-natal, apresentando significância estatística com $p \leq 0,05$. Este resultado concorda com outro realizado no Rio Grande do Sul, que mostra que a maioria das mães que tiveram filhos com BPN apresentaram algum tipo de complicação na gestação, dentre elas, as infecções foram relatadas em 40\% dos casos (FERRAZ e NEVES, 2011).

De acordo com Laurenti et al (2015) a prematuridade, embora não seja considerada como causa básica da morte, vem sendo reconhecida como fator extremamente importante a contribuir para a morte neonatal. Entre os nascidos vivos, o parto prematuro constitui importante causa de morte infantil, principalmente no período neonatal.

O presente estudo demonstra a importância da qualidade do pré-natal, não apenas buscando a realização de todos os exames e acompanhamento preconizados pelos protocolos de assistência à gestante, mas também, ressaltando a importância da capacitação de toda equipe da atenção básica, buscando a captação precoce da gestante para o início do pré-natal e a busca ativa das gestantes que não dão continuidade no processo de cuidado definido para este período.

Os resultados mostram também a importância da detecção precoce das infecções do trato urinário, uma vez que podem resultar em diversas complicações, como o parto prematuro e incidência de baixo peso ao nascer, resultando não somente em problemas para o binômio mãefilho, mas também no alto custo ao sistema de saúde, que terá mais gastos com internamentos, com tratamentos e também com as crescentes taxas de cesáreas realizadas no Brasil.

Julga-se importante também a realização de um estudo mais amplo, uma vez que, os resultados não consideram os casos de gestantes que tiveram acompanhamento pré-natal em clínicas particulares ou que apresentaram falta de continuidade na assistência ofertada pelo SUS.

\section{Conclusão}

O presente estudo permite constatar que existe correlação entre a incidência de infecções do trato urinário durante o período gestacional e o aumento das taxas de prematuridade apresentadas, uma vez que $10,1 \%$ das gestantes incluídas no estudo tiveram ITU, detectadas pela tira reagente no exame de urina I, e que 18,2\% destas gestações resultaram em partos pré-termos. A correlação entre a incidência de ITU e a prematuridade também demonstra a importância na detecção precoce de complicações a fim de evitar, não somente os casos de crianças com baixo 
peso ao nascer, mas também reduzir os índices de mortalidade materno-fetal e os gastos decorrentes que acarretam ao Sistema Único de Saúde.

\section{Referências}

BAGGIO, M.A., PEREIRA, F.C, GUIMARÃES, A,T,B, CALDEIRA, S., VIEIRA, C.S. Programa rede mãe paranaense: análise da atenção pré-natal em uma regional de saúde. Cogitare Enferm. Vol. 21(3):1-10, 2016.

BETTIOL, H., BARBIERI, M.A., SILVA, A.A.M. Epidemiologia do nascimento pré-termo: tendências atuais. Rev Bras Ginecol Obstet. Vol 32(2):57-60, 2010.

BRASIL. Ministério da Saúde. Secretaria de Atenção à Saúde. Departamento de Ações Programáticas Estratégicas. Gestação de alto risco: manual técnico. - 5. ed. - Brasília : Editora do Ministério da Saúde, 2012.302 p. - (Série A. Normas e Manuais Técnicos). Disponível em:< http://bvsms.saude.gov.br/bvs/publicacoes/manual_tecnico_gestacao_alto_risco.pdf $>$. Acesso em: 2 jan. 2017.

COSTA, L.D., CURA, C.C., PERONDI, A.R., FRANÇA, V.F., BORTOLOTI, D.S. Perfil epidemiológico de gestantes de alto risco. Cogitare Enferm. Vol 21(2):1-8, 2016.

D’ADDAZIO, L.B., MORAES, S.R. Microrganismos isolados de infecção do trato urinário da comunidade. Rev Saúde. Vol 4(1):11-13, 2015.

DARZÉ, O.I.S.P., BARROSO, U., LORDELO, M. Preditores clínicos de bacteriúria assintomática na gestação. Rev Bras Ginecol Obstet. Vol 33(8): 196-200, 2011.

DUARTE, G., MARCOLIN, A.C., GONÇALVES, C.V., QUINTANA, S.M., BEREZOWSKI, A.T., NOGUEIRA, A.A., CUNHA, S.P. Infecção Urinária na Gravidez: Análise dos Métodos para Diagnóstico e do Tratamento. Rev Bras Ginecol Obstet. Vol 24(7):471-477, 2002.

DUARTE, G., MARCOLIN, A.C., QUINTANA, S.M, CAVALLI, R.C. Infecção urinária na gravidez. Rev Bras Ginecol Obstet. Vol 30(2):93-100, 2008.

FERRAZ, T.R., NEVES, E.T. Fatores de risco para baixo peso ao nascer em maternidades públicas: um estudo transversal. Rev Gaúcha Enferm. Vol 32(1):86-92, 2011.

FRANCIOTTI, D.L., MAYER, G.N., CANCELIER, A.C.L. Fatores de risco para baixo peso ao nascer: um estudo de caso-controle. Arq Catarinenses de Med. Vol 39(3):63-69, 2010.

GÓMEZ, M.C. Plano de ação para diminuição e controle de infecções urinárias em gestantes na UBS de Nova Contagem I no município de Contagem/MG. 48 f. Monografia (Especialização) Curso de Estratégia de Saúde da Família, Universidade Federal de Minas Gerais, Contagem, 2015. Disponível em: <http://docplayer.com.br/13666548-Plano-de-acao-para-diminuicao-econtrole-de-infeccoes-urinariasem-gestantes-na-ubs-de-nova-contagem-i-no-municipio-decontagem-mg.html>. Acesso em: 26 jul. 2016.

GONÇALVES, Z.R., MONTEIRO, D.L.M. Complicações maternas em gestantes com idade avançada. Rev Femina. Vol 40(5):275-279, 2012. 
GUERRA, G.V.Q.L., SOUZA, A.S.R., COSTA, B.F., NASCIMENTO, F.R.Q., AMARAL, M.A., SERAFIM, A.C.P. Exame simples de urina no diagnóstico de infecção urinária em gestantes de alto risco. Rev Bras Ginecol Obstet. Vol 34(11):488-49, 2012.

LAJOS, G.J. Estudo multicêntrico de investigação em prematuridade no Brasil: implementação, correlação intraclasse e fatores associados à prematuridade espontânea. $227 \mathrm{f}$. Tese (Doutorado) Curso de Saúde Materna e Perinatal, Ciências da Saúde, Faculdade de Ciências Médicas da Universidade Estadual de Campinas - Unicamp, Campinas, 2014. Disponível em: $<$ http://www.bibliotecadigital.unicamp.br/document/?view=000927739>. Acesso em: 2 jan. 2017.

IBGE. Censo Demográfico 2010. Disponível em: <http://www.censo2010.ibge.gov.br>.

Linha Guia da Rede Mãe Paranaense. Paraná. Secretaria de Saúde do Estado do Paraná (SESA). Superintendência de Atenção à Saúde, 2013.

LAURENTI, R., JORGE, M.H.P.M., GOTLIEB, S.L.D., OLIVEIRA, B.Z., PIMENTEL, E.C. O estudo do binômio mãe-filho: descrição e resultados gerais. Rev Bras Epidemiol. Vol 18(2):398$412,2015$.

MACHADO, A.K.F., MARMITT, L.P., CESAR, J.A. Prematuridade tardia no extremo sul do Brasil: um estudo de base populacional. Rev Bras Saúde Materno Infantil. Vol 16(2):113-120, 2016.

MATA, K.S., SANTOS, A.A.P., SILVA, J.M.O., HOLANDA, J.B.L., SILVA, F.C.L. Complicações causadas pela infecção do trato urinário na gestação. Rev Espaço Para A Saúde. Vol 15(4):57-63, 2014.

MOURA, L.B., FERNANDES, M.G. A Incidência de Infecções Urinárias Causadas por E. Coli. Rev Olhar Científico - Faculdades Associadas de Ariquemes. Vol 1(2):411-426, 2010.

NASCIMENTO, W.L.S., OLIVEIRA, F.M., ARAÚJO, G.L.S. Infecção do trato urinário em gestantes usuárias do Sistema Único de Saúde. Ensaios e Ciência: Ciências Biológicas, Agrárias e da Saúde. Vol 16(4):111-123, 2012.

No Dia Mundial da Prematuridade, Secretaria da Saúde ressalta avanços na área. Paraná. Secretaria de Saúde do Estado do Paraná (SESA). Disponível em: $<$ http://www.saude.pr.gov.br/modules/noticias/article.php?storyid=5172)>. Acesso em: 2 dez. 2016.

PAGANOTI C, BITTAR RE, CARVALHO MHB, FRANCISCO RPV, ZUGAIB M. As infecções genitais podem alterar os resultados dos testes preditivos do parto prematuro? Rev Bras Ginecol Obstet. Vol 37(1):10-15, 2015.

PAULA M.L.A., NEGRI, M.M., PAULA, C.L.A., XAVIER, A.R., KANAAN, S., WEIDE, L.C.C. Infecção do trato urinário em mulheres com vida sexual ativa. Jornal Bras Med. Vol 103(2):3741, 2015.

PEDRAZA, D.F., CRISTÓVÃO, F.S. Fatores associados ao peso ao nascer: estudo populacional 
com base nas declarações de nascidos vivos. Arq Ciências Saúde. Vol 22(4):92-99, 2015.

PEDRAZA, D.F. Baixo Peso ao nascer no Brasil: revisão sistemática de estudos baseados no sistema de informações sobre nascidos vivos. Rev Atenção à Saúde. Vol 12(41):37-50, 2014.

Plano estadual de saúde 2016-2019. Secretaria de Saúde do Estado do Paraná (SESA). Paraná. Disponível em: $\quad<$ http://docplayer.com.br/25328043-Plano-estadual-de-saude-documentopreliminar-sujeito-a-revisao-ortografica-e-gramatical-formatacao-editorial-padronizacao-detabelas-e.html>. Acesso em: 2 jan. 2017.

SALCEDO, M.M.B.P., BEITUNE, P., SALIS, M.F., JIMÉNEZ, M.F., AYUB, A.C.K. Como Diagnosticar e Tratar Infecção urinária na gestação. Rev Bras Med. Vol 67(8):270-273, 2010.

SILVA, J.L.C.P., SURITA, F.G.C. Idade materna: resultados perinatais e via de parto. Rev Bras Ginecol Obstet. Vol. 31(7):321-325, 2009.

UNICEF BRASIL [homepage na Internet]. 2013 [acesso em 15 de dezembro de 2016]. Consultoria: pesquisa para estimar a prevalência de nascimentos pré-termo no Brasil e explorar possíveis causas; [aproximadamente 18 telas]. Disponível em: http://www.unicef.org/brazil/pt/br_prematuridade_possiveis_causas.pdf 